\title{
REFLECTION COEFFICIENTS AND ENERGY RATIOS IN A MICROPOLAR THERMOELASTIC MEDIUM WITHOUT ENERGY DISSIPATION
}

\author{
BALJEET SINGH ${ }^{1}$
}

(Received 11 September, 2005; revised 22 June, 2006)

\begin{abstract}
The linear governing equations of a micropolar thermoelastic medium without energy dissipation are solved to show the existence of four plane waves in a two-dimensional model. The expressions for velocities of these plane waves are obtained. The boundary conditions at the free surface are used to obtain a system of four nonhomogeneous equations. These equations are solved numerically for a particular model to obtain reflection coefficients for the incidence of coupled longitudinal displacement and coupled transverse microrotational waves. These reflection coefficients as well as the energy ratios are computed and are shown graphically with the angle of incidence in the presence and absence of thermal effects.
\end{abstract}

2000 Mathematics subject classification: primary 74F05, 74A35.

Keywords and phrases: micropolar thermoelasticity, reflection coefficients, energy ratios.

\section{Introduction}

The dynamical theory of thermoelasticity is the study of interaction between thermal and mechanical fields in solid bodies and is of considerable importance in various engineering fields. The generalized theories of thermoelasticity which admit a finite speed of thermal signals (second sound) have aroused much interest in the last four decades. For example, Lord and Shulman [16], by incorporating a flux-rate term into Fourier's law of heat conduction, formulated a generalized theory which involves a hyperbolic heat transport equation admitting finite speed for thermal signals. Green and Lindsay [12], by including temperature rate among the constitutive variables, developed a temperature-rate-dependent thermoelasticity that does not violate the classical Fourier law of heat conduction, when the body under consideration has a centre of symmetry

\footnotetext{
'Department of Mathematics, Government College, Sector-11, Chandigarh 160 011, India; e-mail: baljeet@networkindia.net.

(C) Australian Mathematical Society 2007, Serial-fee code 1446-181 1/07
} 
and this theory also predicts a finite speed for heat propagation. Chandrasekharaiah [4] referred to this wave-like thermal disturbance as "second sound".

Green and Naghdi [14] established a new thermomechanical theory of deformable media that uses a general entropy balance as postulated in Green and Naghdi [13]. The theory is explained in detail in the context of flow of heat in a rigid solid, with particular reference to the propagation of thermal waves at finite speed. A theory of thermoelasticity for nonpolar bodies, based on the new procedure, was discussed by Green and Naghdi [15]. This theory permits the flow of heat as thermal waves at finite speed, and the heat flow does not involve energy dissipation.

The linear theory of micropolar thermoelasticity was developed by extending the theory of micropolar continua to include thermal effects by Eringen [10] and Nowacki [17]. A generalized theory of linear micropolar thermoelasticity was developed by Boschi and Iesan [3]. Dost and Tabarrok [9] have presented a generalized micropolar thermoelasticity by using Green-Lindsay theory. A theory of micropolar thermoelasticity without energy dissipation which admits the finite speed of heat propagation is due to Ciarletta [8].

Various problems on the propagation of thermal waves at finite speed have been tackled by many authors. For example, Chandrasekharaiah [5-7] studied the propagation of thermoelastic plane waves without energy dissipation. Sinha and Sinha [23] and Sinha and Elsibai [21,22] studied the reflection of thermoelastic waves from the free surface of a solid half-space and at the interface of two semi-infinite media in welded contact, in the context of generalized thermoelasticity. Abd-Alla and AlDawy [1] studied the reflection phenomena of SV waves in a generalized thermoelastic medium. Singh and Kumar [20] and Singh [19] have discussed problems on wave propagation in micropolar thermelasticity in the context of the Lord-Shulman and Green-Lindsay theories.

The present paper is motivated by the theory of micropolar thermoelasticity given by Ciarletta [8]. The paper is organized as follows: In Section 2, the linear governing equations for an isotropic micropolar thermoelastic body without energy dissipation are solved to obtain the expressions for the velocities of four plane waves. Section 3 deals with the reflection phenomena of these plane waves from a stress-free insulated boundary. The last section discusses the numerical part of the problem for a particular model to view the thermal effects on various reflected waves.

\section{Governing equations}

A homogeneous, isotropic, micropolar thermoelastic solid occupying the halfspace is considered in an undisturbed state. It initially has a uniform temperature $T_{0}$. The rectangular Cartesian coordinates are introduced, having the origin on the 
surface $y=0$ and the $y$-axis is chosen in the direction of increasing depth. A twodimensional problem (in the $x y$-plane) is being discussed with the wave front parallel to the $z$-axis.

Following Green and Naghdi [15], Eringen [10] and Ciarletta [8], the constitutive and field equations for an isotropic micropolar thermoelastic medium without energy dissipation and in the absence of body forces and couples become

$$
\begin{gathered}
\sigma_{l j}=\lambda u_{r, r} \delta_{\imath \jmath}+\mu\left(u_{i, j}+u_{j, i}\right)+\kappa\left(u_{j, r}-\epsilon_{i j r} \phi_{r}\right)-\nu \delta_{l j} T, \\
m_{i j}=\alpha \phi_{r, r} \delta_{i j}+\beta \phi_{i, j}+\gamma \phi_{j, i}, \\
(\lambda+\mu) \nabla(\nabla . \mathbf{u})+(\mu+\kappa) \nabla^{2} \mathbf{u}+\kappa \nabla \times \phi-\nu \nabla T=\rho \ddot{\mathbf{u}}, \\
(\alpha+\beta+\gamma) \nabla(\nabla . \phi)-\gamma \nabla \times(\nabla \times \phi)+\kappa \nabla \times \mathbf{u}-2 \kappa \phi=\rho j \ddot{\phi}, \\
K^{*} \nabla^{2} T-\nu T_{0} \nabla \ddot{\mathbf{u}}=\rho C^{*} \ddot{T},
\end{gathered}
$$

where $\lambda, \mu, \kappa, \alpha, \beta, \gamma$ are material constants, $\rho$ is the density, $j$ is the microinertia, $\nu=(3 \lambda+2 \mu+\kappa) \alpha_{t}, \alpha_{t}$ are the coefficients of linear thermal expansion, $C^{*}$ is the specific heat at constant strain and $K^{*}=C^{*}(\lambda+2 \mu) / 4$ is a material constant characteristic of the theory. Here $T(x, y, t)$ is the temperature change above the uniform reference temperature $T_{0}, \mathbf{u}$ is the displacement vector, $\phi$ is the microrotation vector, $t_{i j}$ are the components of force stress and $m_{i j}$ are the components of couple stress. The superposed dots denote the time derivatives.

For the two-dimensional problem, let

$$
\mathbf{u}=\left(\mathbf{u}_{1}, \mathbf{u}_{2}, \mathbf{0}\right) \text { and } \phi=\left(\mathbf{0}, \mathbf{0}, \phi_{3}\right),
$$

where the displacement components $u_{1}$ and $u_{2}$ may be written in terms of the potential functions $q(x, y, t)$ and $\psi(x, y, t)$ as

$$
u_{1}=\frac{\partial q}{\partial x}-\frac{\partial \psi}{\partial y}, \quad u_{2}=\frac{\partial q}{\partial y}+\frac{\partial \psi}{\partial x},
$$

where $\psi=\vec{\psi}_{z}$.

With the help of Equations (2.6) and (2.7), Equations (2.3)-(2.5) reduce to

$$
\begin{aligned}
(\lambda+2 \mu+\kappa) \nabla^{2} q-v T & =\rho \ddot{q}, \\
K^{*} \nabla^{2} T-v T_{0} \nabla^{2} \ddot{q} & =\rho C^{*} \ddot{T}, \\
(\mu+\kappa) \nabla^{2} \psi-\kappa \phi_{3} & =\rho \ddot{\psi}, \\
\gamma \nabla^{2} \phi_{3}+\kappa \nabla^{2} \psi-2 \kappa \phi_{3} & =\rho j \ddot{\phi}_{3} .
\end{aligned}
$$

Equations (2.8) and (2.9) are coupled in $q$ and $T$, whereas Equations (2.10) and (2.11) are coupled in $\phi$ and $\psi$. Solutions of Equations (2.8) to (2.11) are now sought in the form of the harmonic travelling wave

$$
\{q, T, \phi, \psi\}=\{\bar{q}, \bar{T}, \bar{\phi}, \bar{\psi}\} e^{\imath k(x \sin \theta+y \cos \theta-v t)},
$$


in which $v$ is the phase speed, $k$ is the wave number, and $(\sin \theta, \cos \theta)$ denotes the projection of the wave normal onto the $x y$-plane. The homogeneous system of equations in $\bar{q}, \bar{T}, \bar{\phi}$ and $\bar{\psi}$, obtained by inserting (2.12) into (2.8)-(2.11), admits non-trivial solutions and enables us to conclude that there exist four plane waves, namely Coupled Longitudinal Displacement (CLD), Coupled Thermal (CT), Coupled Transverse Displacement (CTD) and Coupled Transverse Microrotational (CTM) waves, with the following distinct velocities:

$$
\begin{aligned}
& v_{1}=V_{\mathrm{CLD}}=\left[(\lambda+2 \mu+\kappa)+d_{1}\right]+\sqrt{\left[(\lambda+2 \mu+\kappa)-d_{1}\right]^{2}+4 e_{1}}, \\
& v_{2}=V_{\mathrm{CT}}=\left[(\lambda+2 \mu+\kappa)+d_{1}\right]-\sqrt{\left[(\lambda+2 \mu+\kappa)-d_{1}\right]^{2}+4 e_{1}}, \\
& v_{3}=V_{\mathrm{CTD}}=\left[(\mu+\kappa)+d_{2}\right]+\sqrt{\left[(\mu+\kappa)-d_{2}\right]^{2}+4 e_{2}}, \\
& v_{4}=V_{\mathrm{CTM}}=\left[(\mu+\kappa)+d_{2}\right]-\sqrt{\left[(\mu+\kappa)-d_{2}\right]^{2}+4 e_{2}},
\end{aligned}
$$

where

$$
d_{1}=\frac{K^{*}}{C^{*}}, \quad d_{2}=\frac{\gamma}{j}+\frac{2 \kappa}{j k^{2}}, \quad e_{1}=\frac{\nu^{2} T_{0}}{C^{*}}, \quad e_{2}=\frac{\kappa^{2}}{j k^{2}}
$$

If the thermal effect is neglected, these four plane waves will reduce to a Longitudinal Displacement wave and two coupled waves as obtained by Parfitt and Eringen [18]. In the next section, the reflection of CLD and CTM waves from a stress-free insulated boundary is considered.

\section{Reflection from a free surface}

In the previous section, it has been discussed that there exist four plane waves in an isotropic micropolar generalized thermoelastic solid without energy dissipation. Any incident wave at the interface of two elastic solid bodies, in general, produces dilatational and rotational waves in both media. Let us now consider an incident CLD or CTM wave (Figure 1). The boundary conditions at the free surface $y=0$ are satisfied if the incident CLD or CTM wave gives rise to reflected CLD, reflected CT, reflected CTD and reflected CTM waves. The surface $y=0$ is free from surface tractions and is assumed to be thermally insulated so that there is no variation of temperature on it. Therefore, the boundary conditions on $y=0$ may be written as

$$
\sigma_{y y}=0, \quad \sigma_{y x}=0, \quad \frac{\partial T}{\partial y}=0, \quad m_{y z}=0 .
$$




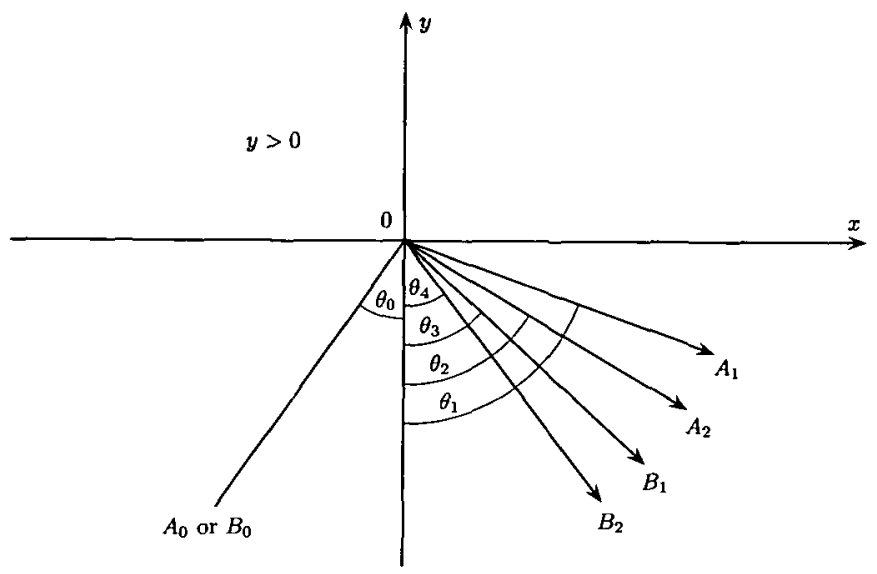

FIGURE 1. Geometry of the problem showing various reflected waves.

The appropriate potentials $q, T, \psi$ and $\phi_{3}$ are taken in the form

$$
\begin{aligned}
q= & \left.A_{0} \exp \left[\iota k_{1}\left(x \sin \theta_{0}+y \cos \theta_{0}\right)-\iota \omega t\right)\right] \\
& +A_{1} \exp \left[\iota k_{1}\left(x \sin \theta_{1}-y \cos \theta_{1}\right)-\iota \omega t\right] \\
& +A_{2} \exp \left[\iota k_{2}\left(x \sin \theta_{2}-y \cos \theta_{2}\right)-\iota \omega t\right], \\
T= & \left.\zeta_{1} A_{0} \exp \left[\iota k_{1}\left(x \sin \theta_{0}+y \cos \theta_{0}\right)-\iota \omega t\right)\right] \\
& +\zeta_{1} A_{1} \exp \left[\iota k_{1}\left(x \sin \theta_{1}-y \cos \theta_{1}\right)-\iota \omega t\right] \\
& +\zeta_{2} A_{2} \exp \left[\iota k_{2}\left(x \sin \theta_{2}-y \cos \theta_{2}\right)-\iota \omega t\right], \\
\psi= & \left.B_{0} \exp \left[\iota k_{4}\left(x \sin \theta_{0}+y \cos \theta_{0}\right)-\iota \omega t\right)\right] \\
& +B_{1} \exp \left[\iota k_{3}\left(x \sin \theta_{3}-y \cos \theta_{3}\right)-\iota \omega t\right] \\
& +B_{2} \exp \left[\iota k_{4}\left(x \sin \theta_{4}-y \cos \theta_{4}\right)-\iota \omega t\right], \\
\phi_{3}= & \left.\eta_{4} B_{0} \exp \left[\iota k_{4}\left(x \sin \theta_{0}+y \cos \theta_{0}\right)-\iota \omega t\right)\right] \\
& +\eta_{3} B_{1} \exp \left[\iota k_{3}\left(x \sin \theta_{3}-y \cos \theta_{3}\right)-\iota \omega t\right] \\
& +\eta_{4} B_{2} \exp \left[\iota k_{4}\left(x \sin \theta_{4}-y \cos \theta_{4}\right)-\iota \omega t\right],
\end{aligned}
$$

where the wave normal of the incident CLD or CTM wave makes angle $\theta_{0}$ with the positive direction of the $y$-axis, and those of the reflected CLD, CT, CTD and CTM waves make $\theta_{1}, \theta_{2}, \theta_{3}$ and $\theta_{4}$ with the same direction, and

$$
\begin{aligned}
\zeta_{i} & =k_{i}^{2}\left[\rho v_{i}^{2}-(\lambda+2 \mu+\kappa)\right] / \nu, & & (i=1,2) \\
\eta_{j} & =k_{j}^{2}\left(\rho v_{j}^{2}-\mu-\kappa\right) / \kappa, & & (j=3,4) .
\end{aligned}
$$

The ratios of the amplitudes of the reflected waves to the amplitude of the incident CLD wave, namely $A_{1} / A_{0}, A_{2} / A_{0}, B_{1} / A_{0}$ and $B_{2} / A_{0}$, give the reflection coefficients 
for reflected CLD, reflected CT, reflected CTD and reflected CTM waves, respectively. Similarly for the incident CTM wave, $A_{1} / B_{0}, A_{2} / B_{0}, B_{1} / B_{0}$ and $B_{2} / B_{0}$ are the reflection coefficients for the reflected CLD, reflected CT, reflected CTD and reflected CTM waves, respectively. The wave numbers $k_{1}, k_{2}, k_{3}, k_{4}$ and the angles $\theta_{1}, \theta_{2}, \theta_{3}, \theta_{4}$ are connected by the relation

$$
k_{1} \sin \theta_{1}=k_{2} \sin \theta_{2}=k_{3} \sin \theta_{3}=k_{4} \sin \theta_{4},
$$

at the surface $y=0$. The relation (3.8) may also be written in order to satisfy the boundary conditions (3.1) as

$$
\frac{\sin \theta_{1}}{v_{1}}=\frac{\sin \theta_{2}}{v_{2}}=\frac{\sin \theta_{3}}{v_{3}}=\frac{\sin \theta_{4}}{v_{4}} .
$$

Using Equations (2.1) and (2.2) and the potentials given by (3.2)-(3.5), the boundary conditions (3.1) result in the following system of four nonhomogeneous equations:

$$
\sum_{j=1}^{4} a_{i j} Z_{j}=b_{i}, \quad i=1, \ldots, 4
$$

where

$$
\begin{aligned}
a_{1 j} & = \begin{cases}{\left[\lambda+(2 \mu+\kappa) \cos ^{2} \theta_{j}+\nu \zeta_{j} / k_{j}{ }^{2}\right]\left(k_{j} / l\right)^{2},} & j=1,2, \\
-(2 \mu+\kappa) \sin \theta_{J} \cos \theta_{j}\left(k_{j} / l\right)^{2}, & j=3,4,\end{cases} \\
a_{2 \jmath} & = \begin{cases}(2 \mu+\kappa) \sin \theta_{j} \cos \theta_{j}\left(k_{j} / l\right)^{2}, & j=1,2, \\
{\left[(\mu+\kappa) \cos ^{2} \theta_{j}-\mu \sin ^{2} \theta_{j}-\kappa \eta_{J} / k_{j}{ }^{2}\right]\left(k_{j} / l\right)^{2},} & j=3,4,\end{cases} \\
a_{3 j} & = \begin{cases}\left(\cos \theta_{j}\right)\left(\zeta_{J} / k_{j}{ }^{2}\right)\left(k_{j} / l\right)^{3}, & j=1,2, \\
0, & j=3,4,\end{cases} \\
a_{4 j} & = \begin{cases}0, & j=1,2, \\
\left(\cos \theta_{j}\right)\left(\eta_{j} / k_{j}{ }^{2}\right)\left(k_{J} / l\right)^{3}, & j=3,4 .\end{cases}
\end{aligned}
$$

For the incident CLD wave, $b_{1}=-a_{11}, b_{2}=a_{21}, b_{3}=a_{31}, b_{4}=a_{41}, l=k_{1}$. For the incident CTM wave, $b_{1}=a_{14}, b_{2}=-a_{24}, b_{3}=a_{34}, b_{4}=a_{44}, l=k_{4}$, and $Z_{1}, Z_{2}$, $Z_{3}, Z_{4}$ are the reflection coefficients of the reflected CLD, CT, CTD and CTM waves, respectively.

Let us consider the energy partition between various reflected waves at a surface element of unit area. The rate of energy transmission at a free surface of a micropolar thermoelastic solid is given by

$$
P^{*}=t_{y y} \dot{u_{2}}+t_{y x} \dot{u_{1}}+m_{y z} \dot{\phi_{3}} .
$$


Following Achenbach [2], for any two complex functions of the form

$$
F=F_{0} \exp \left\{\iota\left(\gamma_{1}-\omega t\right)\right\}, \quad f=f_{0} \exp \left\{\iota\left(\gamma_{2}-\omega t\right)\right\}
$$

where $F_{0}$ and $f_{0}$ are real-valued functions, the time average of the product of the real parts of the two complex functions is given by

$$
\langle R(F) \times R(f)\rangle=\frac{1}{2} R(F \bar{f}),
$$

where $\bar{f}$ is the complex conjugate of $f$.

The energy ratios of the reflected waves are obtained by calculating the ratio of $P^{*}$ for the reflected waves to $P^{*}$ for the incident wave. Substituting Equations (2.1), (2.2), (2.7), (3.12) and (3.13) into Equation (3.11), we obtained the $P^{*}$ for various incident and reflected waves and hence the energy ratios. The following expressions of energy ratios $E_{i}(i=1, \ldots, 4)$ for various reflected waves are obtained:

(i) For the incident CLD wave

$$
\begin{aligned}
& E_{1}=Z_{1}{ }^{2}, \\
& E_{2}=\left[\frac{\lambda+2 \mu+\kappa+v \zeta_{2} / k_{2}{ }^{2}}{\lambda+2 \mu+\kappa+v \zeta_{1} / k_{1}{ }^{2}}\right]\left(\frac{\cos \theta_{2}}{\cos \theta_{0}}\right)\left(\frac{k_{2}}{k_{1}}\right)^{3} Z_{2}{ }^{2}, \\
& E_{3}=\left[\frac{\mu+\kappa-\left(\eta_{3} / k_{3}{ }^{2}\right)\left(\gamma \eta_{3}+\kappa\right)}{\lambda+2 \mu+\kappa+v \zeta_{1} / k_{1}{ }^{2}}\right]\left(\frac{\cos \theta_{3}}{\cos \theta_{0}}\right)\left(\frac{k_{3}}{k_{1}}\right)^{3} Z_{3}{ }^{2}, \\
& E_{4}=\left[\frac{\mu+\kappa-\left(\eta_{4} / k_{4}{ }^{2}\right)\left(\gamma \eta_{4}+\kappa\right)}{\lambda+2 \mu+\kappa+\nu \zeta_{1} / k_{1}{ }^{2}}\right]\left(\frac{\cos \theta_{4}}{\cos \theta_{0}}\right)\left(\frac{k_{4}}{k_{1}}\right)^{3} Z_{4}{ }^{2} .
\end{aligned}
$$

(ii) For the incident CTM wave

$$
\begin{aligned}
& E_{1}=\left[\frac{\lambda+2 \mu+\kappa+\nu \zeta_{1} / k_{1}{ }^{2}}{\mu+\kappa-\left(\eta_{4} / k_{4}{ }^{2}\right)\left(\gamma \eta_{4}+\kappa\right)}\right]\left(\frac{\cos \theta_{1}}{\cos \theta_{0}}\right)\left(\frac{k_{1}}{k_{4}}\right)^{3} Z_{1}{ }^{2}, \\
& E_{2}=\left[\frac{\lambda+2 \mu+\kappa+\nu \zeta_{2} / k_{2}{ }^{2}}{\mu+\kappa-\left(\eta_{4} /{k_{4}}^{2}\right)\left(\gamma \eta_{4}+\kappa\right)}\right]\left(\frac{\cos \theta_{2}}{\cos \theta_{0}}\right)\left(\frac{k_{2}}{k_{4}}\right)^{3} Z_{2}{ }^{2}, \\
& E_{3}=\left[\frac{\mu+\kappa-\left(\eta_{3} /{k_{3}}^{2}\right)\left(\gamma \eta_{3}+\kappa\right)}{\mu+\kappa-\left(\eta_{4} /{k_{4}}^{2}\right)\left(\gamma \eta_{4}+\kappa\right)}\right]\left(\frac{\cos \theta_{3}}{\cos \theta_{0}}\right)\left(\frac{k_{3}}{k_{4}}\right)^{3} Z_{3}{ }^{2}, \\
& E_{4}=Z_{4}{ }^{2}
\end{aligned}
$$

Here the energy ratios $E_{1}, E_{2}, E_{3}$ and $E_{4}$ correspond to the reflected CLD, CT, CTD and CTM waves, respectively. 


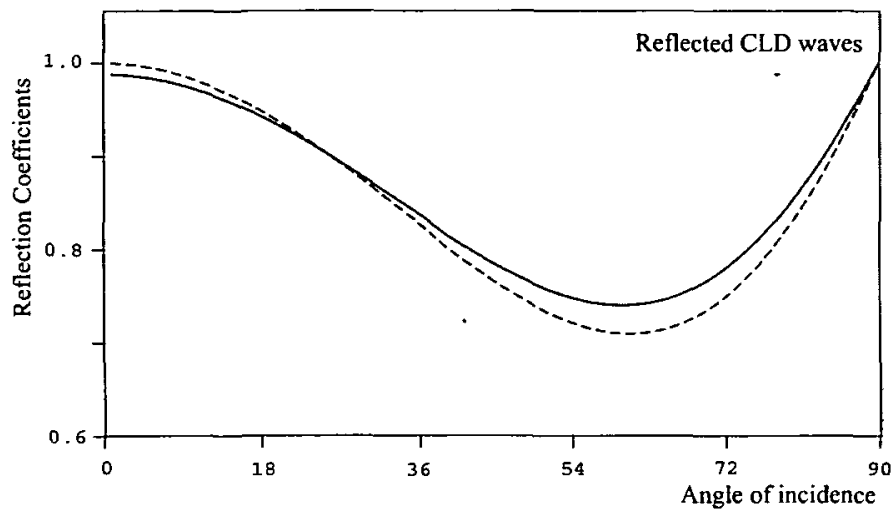

FIGURE 2. Reflection coefficients of reflected CLD waves for incidence of CLD wave.

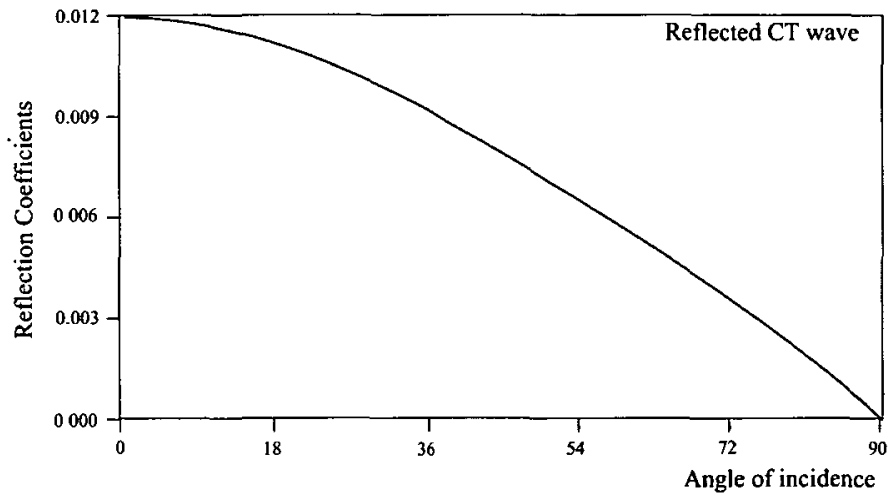

FIGURE 3. Reflection coefficients of reflected CT waves for incidence of CLD wave.

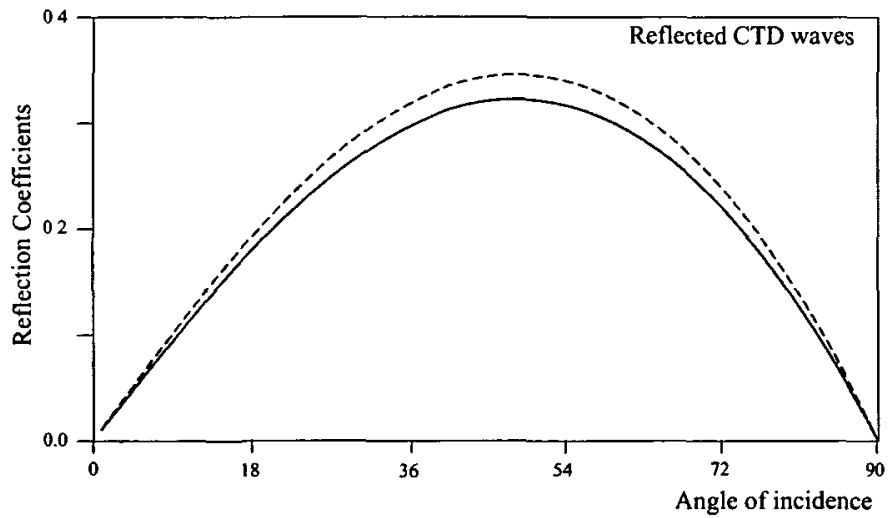

FIGURE 4. Reflection coefficients of reflected CTD waves for incidence of CLD wave. 


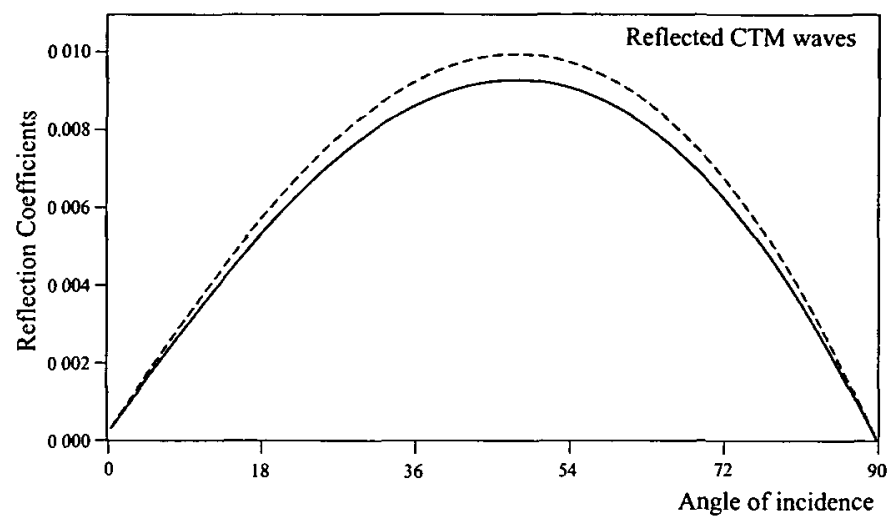

FIGURE 5. Reflection coefficients of reflected CTM waves for incidence of CLD wave.

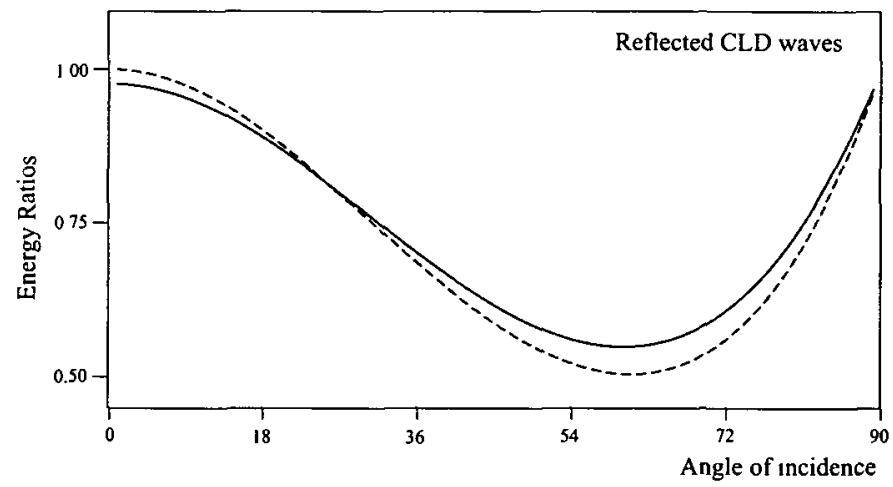

FIGURE 6. Energy ratios of reflected CLD waves for incidence of CLD wave.

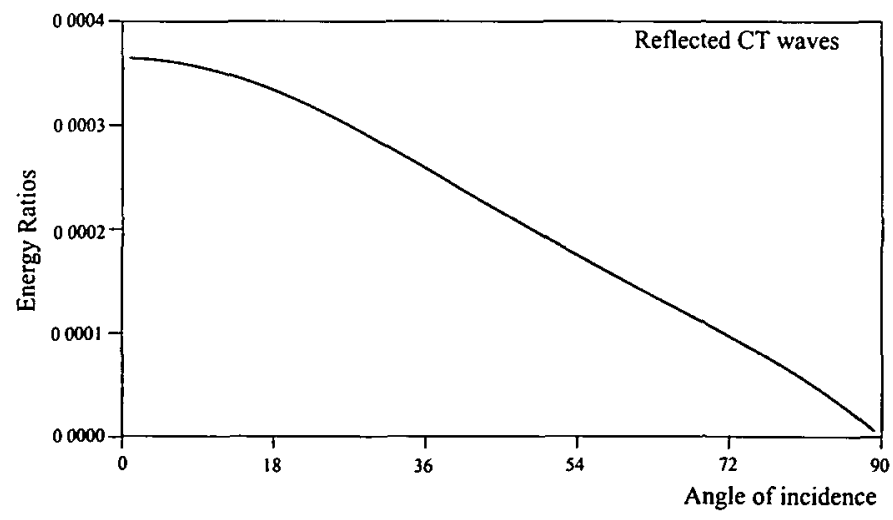

FIGURE 7. Energy ratios of reflected CT waves for incidence of CLD wave. 


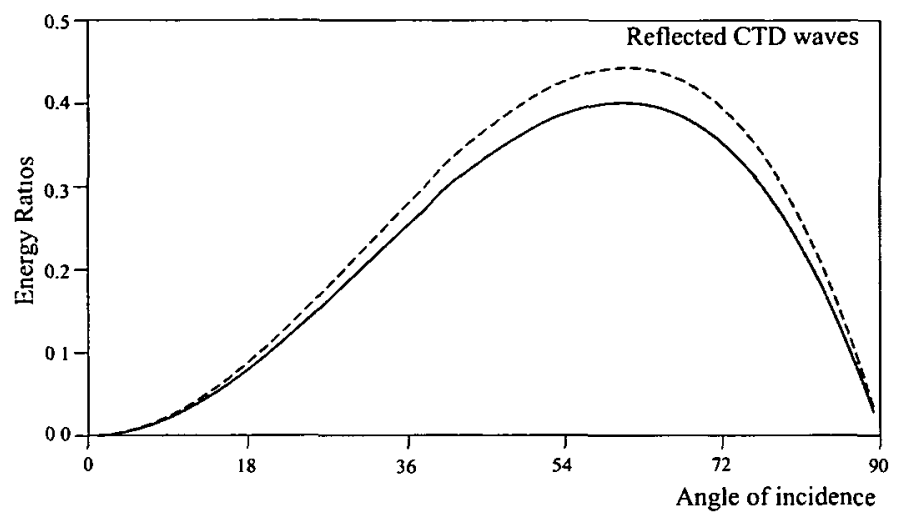

FIGURE 8. Energy ratios of reflected CTD waves for incidence of CLD wave.

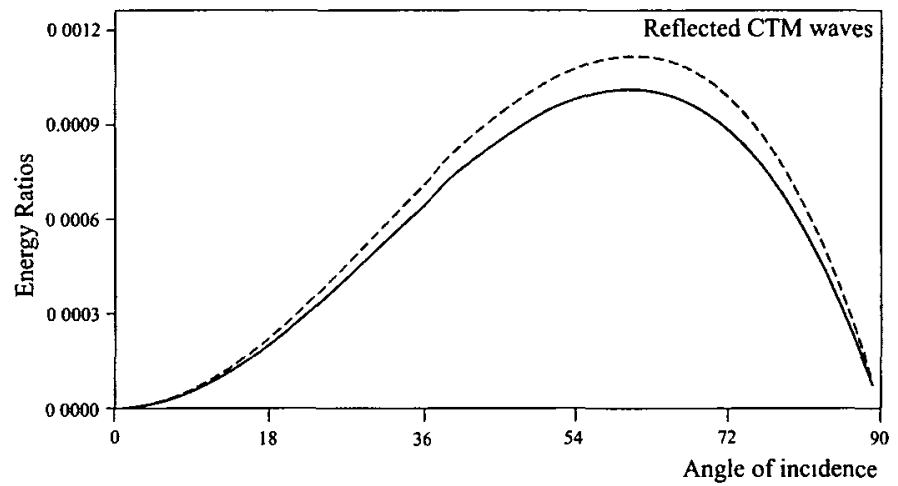

FIGURE 9. Energy ratios of reflected CTM waves for incidence of CLD wave.

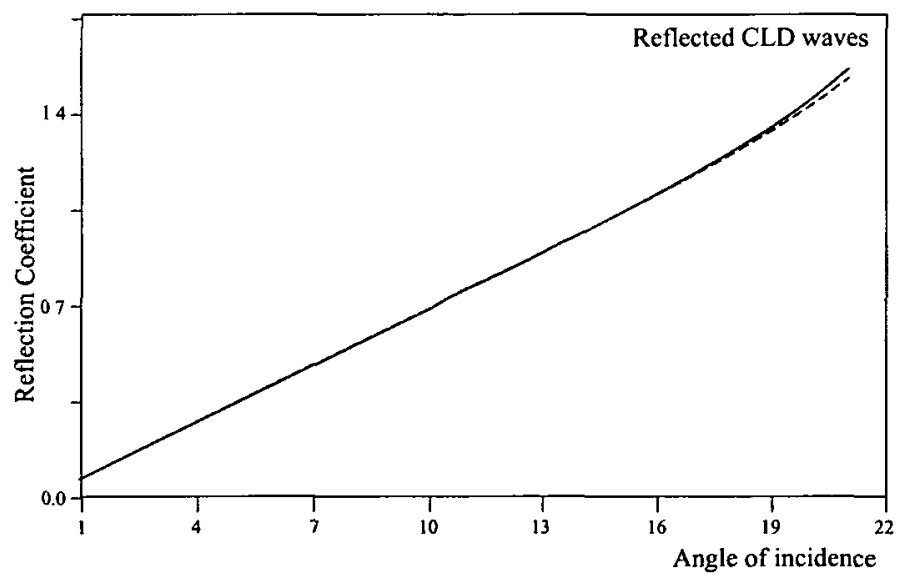

FIGURE 10. Reflection coefficients of reflected CLD waves for incidence of CTM wave. 
[11] Reflection coefficients and energy ratios in a micropolar thermoelastic medium

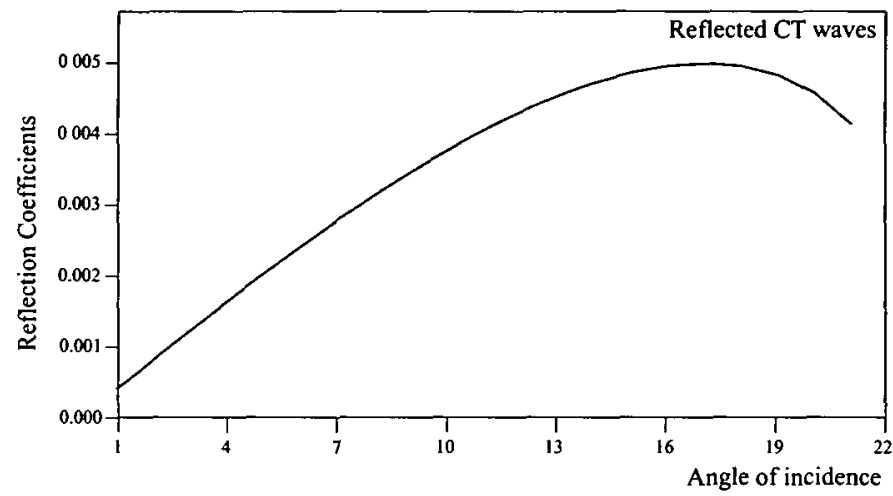

FIGURE 11. Reflection coefficients of reflected CT waves for incidence of CTM wave.

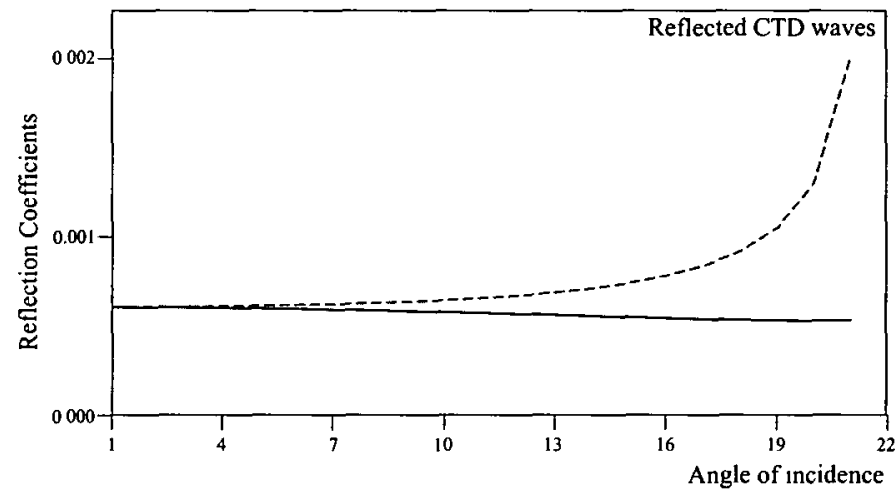

FIGURE 12. Reflection coefficients of reflected CTD waves for incidence of CTM wave.

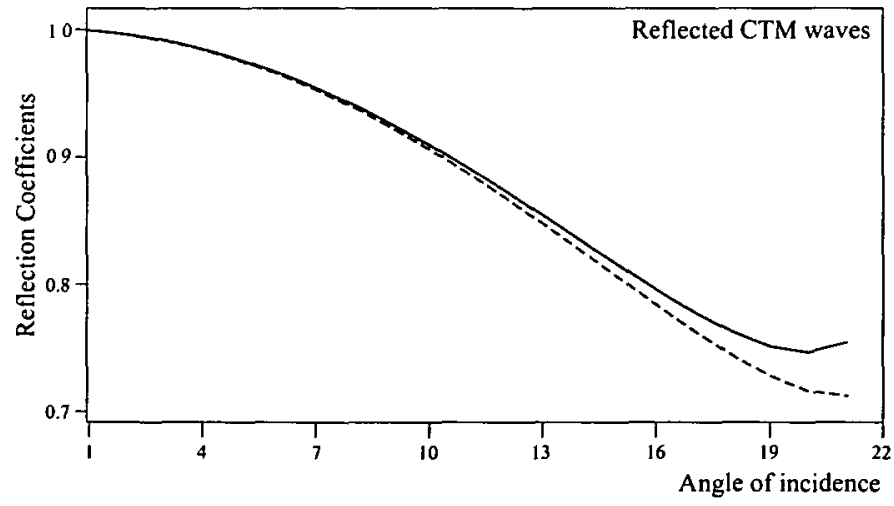

FIGURE 13. Reflection coefficients of reflected CTM waves for incidence of CTM wave. 


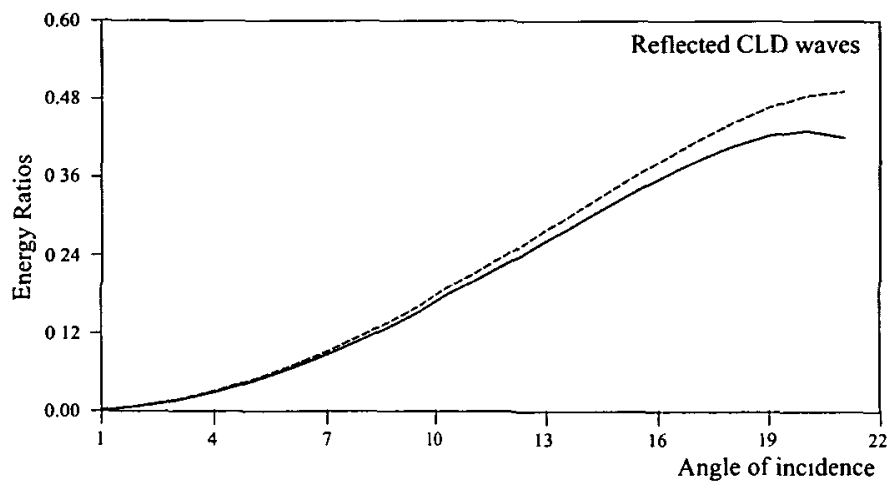

FIGURE 14. Energy ratios of reflected CLD waves for incidence of CTM wave.

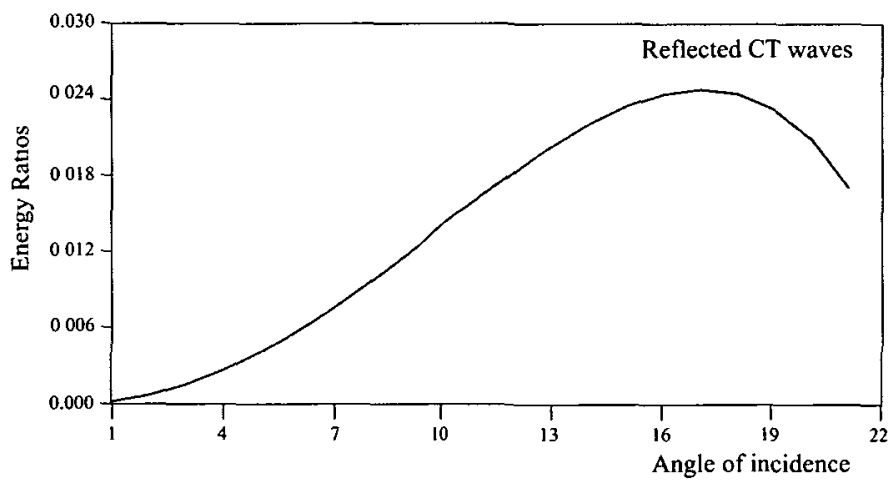

FIGURE 15. Energy ratios of reflected CT waves for incidence of CTM wave.

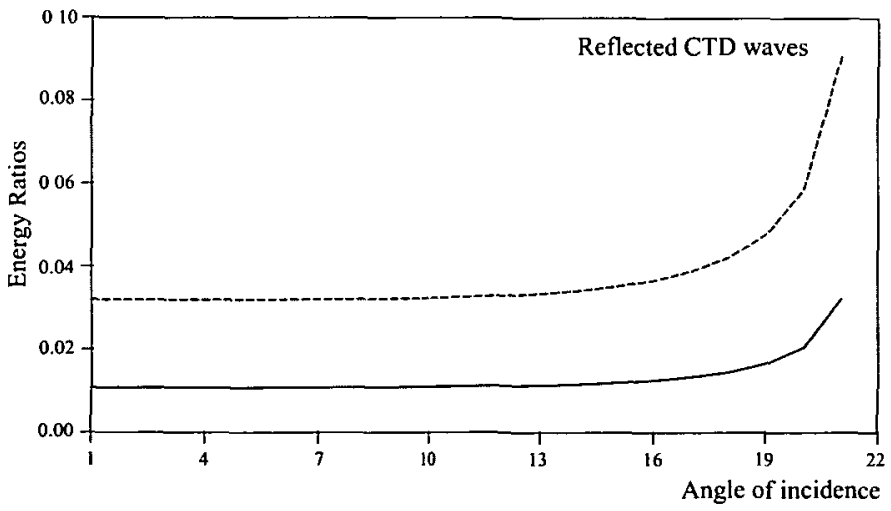

FIGURE 16. Energy ratios of reflected CTD waves for incidence of CTM wave. 


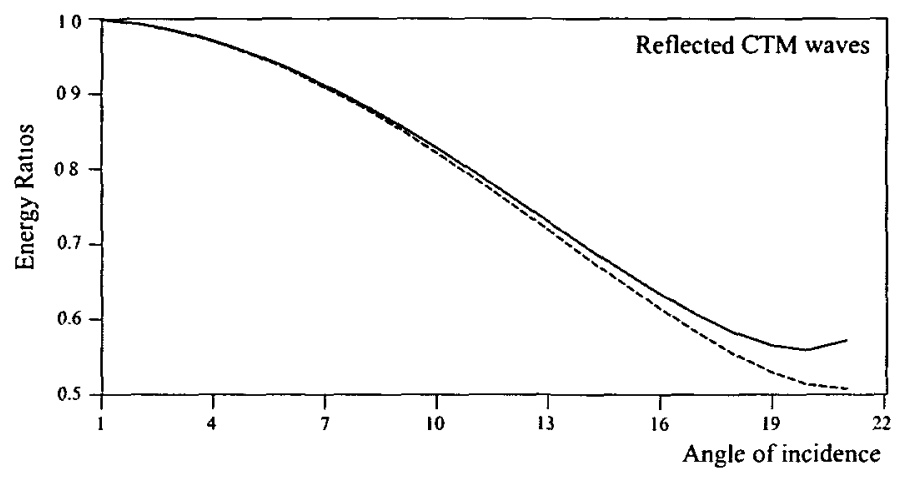

FIGURE 17. Energy ratios of reflected CTM waves for incidence of CTM wave.

\section{Numerical results and discussion}

Following Gauthier [11], the physical constants for a micropolar thermoelastic solid are considered to be $\lambda=7.59 \times 10^{11} \mathrm{dyne} / \mathrm{cm}^{2}, \mu=1.89 \times 10^{11} \mathrm{dyne} / \mathrm{cm}^{2}$, $\kappa=0.0149 \times 10^{11} \mathrm{dyne} / \mathrm{cm}^{2}, \gamma=0.268 \times 10^{11} \mathrm{dyne}, C^{*}=0.23 \mathrm{cal} / \mathrm{g}^{\circ} \mathrm{C}, v=0.3$, $j=0.0196 \mathrm{~cm}^{2}, \rho=2.19 \mathrm{gm} / \mathrm{cm}^{3}, j k^{2}=0.1$.

Making use of Equations (2.13) to (2.17) and (3.6) to (3.9), the system of equations (3.10) is solved numerically by the Gauss elimination method and the reflection coefficients of various reflected waves are obtained. The energy ratios $E_{\imath}$ ( $i=$ $1, \ldots, 4)$ of the reflected waves are also computed for the incidence of both the coupled longitudinal displacement and the coupled transverse microrotational waves. The reflection coefficients and energy ratios are shown graphically with the angle of incidence of the striking wave. The dotted curve in Figures 2-17 represent the variations in the absence of thermal disturbances.

(a) Incidence CLD wave. The reflection coefficients and energy ratios for reflected CLD waves first decrease to their minimum values and then increase to their maximum values. The reflection coefficients and energy ratios of the reflected CLD waves are shown graphically in Figures 2 and 6, respectively. The deviation of the solid curve from the dotted curve in these figures shows the thermal effect on the reflection coefficient and the energy ratio of the reflected CLD wave at a particular angle of incidence.

The reflection coefficient and energy ratio for reflected coupled thermal waves decrease with the increase in the angle of incidence. The dotted curve does not appear in Figures 3 and 7 due to the absence of thermal disturbances.

The reflection coefficients and energy ratios for the reflected CTD and CTM waves increase from minimum to maximum and then decrease to their respective minimum values. The effect of thermal disturbances is observed at a maximum near peaks and at 
a minimum at normal and grazing incidence. These reflection coefficients and energy ratios are shown graphically in Figures 4 and 5 and Figures 8 and 9.

(b) Incidence CTM wave. The reflection coefficients and energy ratios for reflected waves are shown graphically with the angle of incidence varying from $1^{\circ}$ to $21^{\circ}$, as there is no reflected wave beyond the critical angle $21^{\circ}$. The reflection coefficients and energy ratios for the reflected CLD waves increase with the increase in the angle of incidence, as shown in Figures 10 and 14. The effect of thermal disturbance on the reflection coefficient and energy ratio of the reflected CLD wave is less considerable.

The reflection coefficients and energy ratios for reflected coupled thermal waves are shown in Figures 11 and 15. The dotted curves in these figures disappear due to the absence of thermal disturbance. The solid curve variation in Figure 15 is shown after multiplying its original value by $10^{3}$.

The reflection coefficients and energy ratios of the reflected CTD waves are shown graphically in Figures 12 and 16, respectively. The comparison of solid and dotted curves in these figures shows the impact of thermal disturbances on the reflected CTD waves. The solid and dotted curve variations in Figure 16 are shown after multiplying their original values by $10^{3}$ and $10^{9}$, respectively.

The reflection coefficients and energy ratios for reflected CTM waves decrease with the increase in the angle of incidence. The maximum thermal effect on the reflection coefficient and the energy ratio is observed near the critical angle and $s$ at a minimum near normal incidence. These variations are shown graphically in Figures 13 and 17.

\section{Conclusions}

The field equations of micropolar thermoelasticity without energy dissipation are solved for a two-dimensional model in the $x y$-plane. The four plane waves are found to propagate with distinct speeds. The reflection of coupled longitudinal displacement and coupled transverse microrotational waves from a thermally insulated free surface is considered. The boundary conditions at the free surface are used to obtain a system of four equations in the reflection coefficients of various reflected waves. The energy ratios are calculated for the incidence of both striking waves. These reflection coefficients and energy ratios are also computed numerically for a particular material. The thermal disturbances in the micropolar material affect significantly the reflection coefficients and the energy ratios of various reflected waves.

\section{Acknowledgements}

The author is grateful to the Indian National Science Academy, New Delhi for INSA Young Scientist Project (No. BS/YSP/2005 1489). 


\section{References}

[1] A. N. Abd-Alla and Al-Dawy, "The reflection phenomena of SV waves in a generalized thermoelastic medium", Int. J. Math. Math. Sci. 23 (2000) 529-546.

[2] J. D. Achenbach, Wave Propagation in Elastic Solids (North Holland, Amsterdam, 1973).

[3] E. Boschi and D. Iesan, "A generalized theory of linear micropolar thermoelasticity", Meccanica 8 (1973) 154-157.

[4] D. S. Chandrasekharaiah, "Thermoelasticity with second sound: A review", Appl. Mech. Rev. 39 (1986) 355-376.

[5] D. S. Chandrasekharaiah, "One dimensional waves in thermoelastic half-space without energy dissipation", Int. J. Engng. Sci. 13 (1996) 1447-1455.

[6] D. S. Chandrasekharaiah, "Thermoelastic plane waves without energy dissipation", Mech. Res. Commun. 23 (1996) 549-555.

[7] D. S. Chandrasekharaiah, "Complete solutions in the theory of thermoelasticity without energy dissipation", Mech. Res. Commun. 24 (1997) 625-630.

[8] M. Ciarletta, "A theory of micropolar thermoelasticity without energy dissipation", J. Thermal Stresses 22 (1999) 581-594.

[9] S. Dost and B. Tabarrok, "Generalized micropolar thermoelasticity", Int. J. Engng. Sci. 16 (1978) 173-183.

[10] A. C. Eringen, Foundations of Micropolar Thermoelasticity, International Centre for Mechanical Science, Courses and Lectures 23 (Springer, Berlin, 1970).

[11] R. D. Gauthier, "Experimental investigation on micropolar media", in Mechanics of Micropolar Media (eds. O. Brulin and R. K. T. Hsieh), (World Scientific, Singapore, 1982).

[12] A. E. Green and K. A. Lindsay, "Thermoelastıcity", J. Elasticity 2 (1972) 1-7.

[13] A. E. Green and P. M. Naghdi, "On thermodynamics and the nature of the second law", Proc. Roy. Soc. London A 357 (1977) 253-270.

[14] A. E. Green and P. M. Naghdi, "A re-examination of the basic postulates of thermomechanics", Proc. Roy. Soc. London A 432 (1991) 171-194.

[15] A. E. Green and P. M. Naghdi, "Thermoelasticity without energy dissipation", J. Elasticity 31 (1993) 189-209.

[16] H. W. Lord and Y. Shulman, "A generalized dynamical theory of thermoelasticity", J. Mech. Phys. Solids 15 (1967) 209-309.

[17] W. Nowacki, Theory of Asymmetric Elasticity (Pergamon, Oxford, 1986).

$[18] \cdot V$. R. Parfitt and A. C. Eringen, "Reflection of plane waves from a flat boundary of a micropolar elastic half-space", J. Acoust. Soc. Am. 45 (1969) 1258-1272.

[19] B. Singh, "Reflection of plane sound wave from a micropolar generalized thermoelastic solid half-space", J. Sound Vibration 235 (2000) 685-696.

[20] B. Singh and R. Kumar, "Reflection of plane waves from a flat boundary of a micropolar generalized thermoelastic half-space", Int. J. Engng. Sci. 36 (1998) 865-890.

[21] A. N. Sinha and K. A. Elsibai, "Reflection of thermoelastic waves at a solid half-space with two relaxation times", J. Thermal Stresses 19 (1996) 763-777.

[22] A. N. Sinha and K. A. Elsibai, "Reflection and refraction of thermoelastic waves at an interface of two semi-infinite media with two relaxation times", J. Thermal Stresses 20 (1997) 129-146.

[23] A. N. Sinha and S. B. Sinha, "Reflection of thermoelastic waves at a solid half-space with therma] relaxation", J. Phys. Earth 22 (1974) 237-244. 